\title{
Reviewer Acknowledgement (2020)
}

๑) Springer-Verlag GmbH Germany, part of Springer Nature 2021

Distributed Computing relies on generous contributions of time and expertise by scholars who agree to review articles on a voluntary basis. The Editor-in-Chief would like to credit the following peer reviewers who completed reviews for the journal over the course of 2020:

$\begin{array}{lll}\text { Dan Alistarh } & \text { Mohsen Ghaffari } & \text { Dennis Olivetti } \\ \text { Emmanuelle Anceaume } & \text { Sukumar Ghosh } & \text { Fukuhito Ooshita } \\ \text { Guy Avni } & \text { George Giakkoupis } & \text { Aurojit Panda } \\ \text { Leonid Barenboim } & \text { Kerim Gokarslan } & \text { Gopal Pandurangan } \\ \text { Ruben Becker } & \text { Guy Golan-Gueta } & \text { Boaz Patt-Shamir } \\ \text { Gregory M. Bodwin } & \text { Danny Hendler } & \text { Paolo Penna } \\ \text { Benedikt Bollig } & \text { Maurice Herlihy } & \text { Seth Pettie } \\ \text { Borzoo Bonakdarpour } & \text { D Ellis Hershkowitz } & \text { Mikaël Rabie } \\ \text { Trevor Brown } & \text { Taisuke Izumi } & \text { Anisur Rahaman Molla } \\ \text { Janna Burman } & \text { Colette Johnen } & \text { Nicolas Rivera } \\ \text { Keren Censor-Hillel } & \text { Panagiotis Katsaros } & \text { Peter Robinson } \\ \text { Yi-Jun Chang } & \text { Felix Klaedtke } & \text { William Rosenbaum } \\ \text { Natalia Chechina } & \text { Rastislav Královič } & \text { Konstantinos Sagonas } \\ \text { Andrea Clementi } & \text { Danny Krizanc } & \text { Ulrich Schmid } \\ \text { Anne Condon } & \text { Akash Kumar } & \text { Milad Sefidgaran } \\ \text { Gennaro Cordasco } & \text { Christoph Lenzen } & \text { Eric Severson } \\ \text { Sandro Coretti } & \text { Jean-Philippe Martin } & \text { Yuichi Sudo } \\ \text { Luis Cruz-Filipe } & \text { Yannic Maus } & \text { Jukka Suomela } \\ \text { Carole Delporte-Gallet } & \text { Avery Miller } & \text { Adriana Szekeres } \\ \text { Giorgio Delzanno } & \text { Neeraj Mittal } & \text { Gadi Taubenfeld } \\ \text { Giuseppe Di Luna } & \text { Pedro Montealegre } & \text { Emilio Tuosto } \\ \text { Rayna Dimitrova } & \text { Adam Morrison } & \text { Yadu Vasudev } \\ \text { Michal Dory } & \text { William Moses Jr. } & \text { Roman Vitenberg } \\ \text { Clemens Dubslaff } & \text { Achour Mostefaoui } & \text { Philipp Woelfel } \\ \text { Laurent Feuilloley } & \text { Shuai Mu } & \text { Yaoqing Yang } \\ \text { Adrian Francalanza } & \text { Sagnik Mukhopadhyay } & \text { Viktor Zamaraev } \\ \text { Vijay Garg } & \text { Ofer Neiman } & \text { Leqi Zhu } \\ \text { Rati Gelashvili } & \text { Nicolas Nisse } & \\ & & \end{array}$

Publisher's Note Springer Nature remains neutral with regard to jurisdictional claims in published maps and institutional affiliations. 